\title{
On the maximum number of maximum independent sets
}

\author{
E. Mohr \\ D. Rautenbach \\ Institut für Optimierung und Operations Research, Universität Ulm, Ulm, Germany \\ \{elena.mohr, dieter.rautenbach\}@uni-ulm.de
}

\begin{abstract}
We give a very short and simple proof of Zykov's generalization of Turán's theorem, which implies that the number of maximum independent sets of a graph of order $n$ and independence number $\alpha$ with $\alpha<n$ is at most $\left\lceil\frac{n}{\alpha}\right\rceil^{n \bmod \alpha}\left\lfloor\frac{n}{\alpha}\right\rfloor^{\alpha-(n \bmod \alpha)}$. Generalizing a result of Zito, we show that the number of maximum independent sets of a tree of order $n$ and independence number $\alpha$ is at most $2^{n-\alpha-1}+1$, if $2 \alpha=n$, and, $2^{n-\alpha-1}$, if $2 \alpha>n$, and we also characterize the extremal graphs. Finally, we show that the number of maximum independent sets of a subcubic tree of order $n$ and independence number $\alpha$ is at most $\left(\frac{1+\sqrt{5}}{2}\right)^{2 n-3 \alpha+1}$, and we provide more precise results for extremal values of $\alpha$.
\end{abstract}

Keywords: Turán graph; tree; independence number; maximum independent set; Fibonacci number 


\section{Introduction}

We consider only finite, simple, and undirected graphs, and use standard terminology and notation. An independent set in a graph $G$ is a set of pairwise non-adjacent vertices of $G$. The independence number $\alpha(G)$ of $G$ is the maximum cardinality of an independent set in $G$. An independent set in $G$ is maximal if no proper superset is an independent set in $G$, and maximum if it has cardinality $\alpha(G)$. For a graph $G$, let $\sharp \alpha(G)$ be the number of maximum independent sets in $G$.

In the present paper we study the maximum number of maximum independent sets as a function of the order and the independence number in general graphs, trees, and subcubic trees. Before we come to our results, we mention some related research.

For a tree $T$ of order $n>1$, Zito [12] showed

$$
\sharp \alpha(T) \leq \begin{cases}2^{\frac{n-2}{2}}+1 & , \text { if } n \text { is even, and } \\ 2^{\frac{n-3}{2}} & , \text { if } n \text { is odd. }\end{cases}
$$

Since $\alpha(T) \geq n / 2$, it is not difficult to show that (1) implies

$$
\sharp \alpha(T) \leq 2^{\alpha(T)-1}+1,
$$

cf. [2] for a simple independent proof. For similar results concerning the maximum number of maximal independent sets see [6, 11].

Jou and Chang [5] observed that Moon and Moser's [7] result on the maximum number of maximal independent sets implies

$$
\sharp \alpha(G) \leq\left\{\begin{array}{lll}
3^{\frac{n}{3}} & , \text { if } n \bmod 3=0, \\
4 \cdot 3^{\frac{n-4}{3}} & , \text { if } n \bmod 3=1, \text { and } \\
2 \cdot 3^{\frac{n-2}{3}} & , \text { if } n \bmod 3=2,
\end{array}\right.
$$

for every graph $G$ of order $n$. This is actually an immediate consequence of Zykov's generalization [13] of Turán's theorem [10]; independently shown also by Roman [9]. For positive integers $n$ and $p$, let $T_{p}(n)$ be the complete $p$-partite graph with $n \bmod p$ partite sets of order $\left\lceil\frac{n}{p}\right\rceil$ and $p-(n \bmod p)$ partite sets of order $\left\lfloor\frac{n}{p}\right\rfloor$, that is, $T_{p}(n)$ is the Turán graph. A clique in a graph $G$ is a set of pairwise adjacent vertices of $G$. For a graph $G$ and a positive integer $q$, let $\sharp \omega^{(p)}(G)$ be the number of cliques of order $p$ in $G$.

Theorem 1 (Zykov [13]). Let $n, q$, and $p$ be integers with $2 \leq q<p \leq n$. If $G$ is a graph of order $n$ with no clique of order $p$, then $\sharp \omega^{(q)}(G) \leq \sharp \omega^{(q)}\left(T_{p-1}(n)\right)$ with equality if and only if $G=T_{p-1}(n)$.

As our first contribution, we give a very short and simple proof of Theorem 1 inspired by the 5th proof from The Book [1] of Turán's theorem. Applying the special case $q=p-1$ of Theorem 1 to the complement $\bar{G}$ of a graph $G$ immediately implies the following.

Corollary 2. If $G$ is a graph of order $n$ and independence number $\alpha$ with $\alpha<n$, then

$$
\sharp \alpha(G) \leq\left\lceil\frac{n}{\alpha}\right\rceil^{n \bmod \alpha}\left\lfloor\frac{n}{\alpha}\right\rfloor^{\alpha-(n \bmod \alpha)} .
$$

\{theorem

Furthermore, equality holds in (3) if and only if $G$ is the complement of $T_{\alpha}(n)$.

Corollary 2 also follows from a result of Nielsen [8] who showed that the right hand side of (3) is a tight upper bound on the number of maximal independent sets of cardinality exactly $\alpha$ for every graph $G$ of order $n$ regardless of the independence number of $G$.

Our further results concern trees and subcubic trees.

The next result is a common generalization of (1) and (2). 
Theorem 3. If $T$ is a tree of order $n$ and independence number $\alpha$, then

$$
\sharp \alpha(T) \leq \begin{cases}2^{n-\alpha-1}+1 & , \text { if } 2 \alpha=n, \text { and } \\ 2^{n-\alpha-1} & , \text { if } 2 \alpha>n .\end{cases}
$$

Furthermore, equality holds in (4) if and only if $T$ arises by subdividing $n-\alpha-1$ edges of $K_{1, \alpha}$ once.

As it turns out, the maximum number of maximum independent sets in subcubic trees is closely related to the famous Fibonacci numbers. Let $f(n)$ denote the $n$-th Fibonacci number, that is,

$$
f(n)= \begin{cases}0 & , \text { if } n=0, \\ 1 & \text {, if } n=1, \\ f(n-1)+f(n-2) & , \text { if } n \geq 2 .\end{cases}
$$

Our first result for subcubic trees concerns the smallest possible value of the independence number in (subcubic) trees. For a positive integer $k$, let $T(k)$ arise by attaching a new endvertex to every vertex of a path of order $k$. Since

$$
\begin{aligned}
& \sharp \alpha(T(1))=2, \\
& \sharp \alpha(T(2))=3, \text { and } \\
& \sharp \alpha(T(k))=\sharp \alpha(T(k-1))+\sharp \alpha(T(k-2)) \text { for every } k \geq 3,
\end{aligned}
$$

we obtain

$$
\sharp \alpha(T(k))=f(k+2)
$$

for every positive integer $k$.

Theorem 4. If $T$ is a subcubic tree of order $n$ and independence number $\alpha=\frac{n}{2}$, then

$$
\sharp \alpha(T) \leq f(\alpha+2)
$$

Furthermore, equality holds in (5) if and only if $T=T(\alpha)$.

Our second result for subcubic trees concerns the largest possible value of the independence number in subcubic trees. If $T$ is a tree, then $T^{\prime}$ arises from $T$ by attaching a $P_{3}$ if $V(T)$ is the disjoint union of $V(T)$ and $\{x, y, z\}$, and $E(T)=E\left(T^{\prime}\right) \cup\{u y, x y, y z\}$, where $u$ is some vertex of $T$.

Theorem 5. If $T$ is a subcubic tree of order $n$ and independence number $\alpha$, then

$$
\alpha(T) \leq \frac{2 n+1}{3} .
$$

Furthermore, equality holds in (6) if and only if $T$ arises from $K_{1}$ by iteratively attaching $P_{3} s$, in which case $\sharp \alpha(T)=1$.

For given positive integers $n$ and $\alpha$ with $\alpha \leq \frac{2 n+1}{3}$, suitably combining the extremal trees from Theorem 4 and Theorem 5 allows to construct subcubic trees with order $n$ and independence number $\alpha$ that satisfy

$$
\sharp \alpha(T)=\Omega(f(2 n-3 \alpha+1)) .
$$

This implies that our last result for subcubic trees is best possible up to small constant factors and additive terms.

Theorem 6. If $T$ is a subcubic tree of order $n$ and independence number $\alpha$, then

$$
\sharp \alpha(T) \leq\left(\frac{1+\sqrt{5}}{2}\right)^{2 n-3 \alpha+1} .
$$

All proofs are give in the next section. 


\section{Proofs}

Proof of Theorem 1, Let $G$ be a graph of order $n$ with no clique of order $p$ that maximizes $\sharp \omega^{(q)}(G)$. Let $G_{0}$ arise from $G$ by removing all edges that do not belong to a clique of order $q$ in $G$. Clearly, $G_{0}$ has no clique of order $p$, and $\sharp \omega^{(q)}\left(G_{0}\right)=\sharp \omega^{(q)}(G)$.

Claim 1. $G_{0}$ is a complete multipartite graph.

Proof of Claim 1. Suppose, for a contradiction, that the claim fails. This implies the existence of three vertices $u, v$, and $w$ such that $u$ is not adjacent to $v$ or $w$, but $v$ and $w$ are adjacent. Let $d^{(q)}(u)$ be the number of cliques of order $q$ in $G_{0}$ that contain $u$, that is, $d^{(q)}(u)=\sharp \omega^{(q-1)}\left(G_{0}\left[N_{G_{0}}(u)\right]\right)$. Let $d^{(q)}(v)$ and $d^{(q)}(w)$ be defined analogously. If $d^{(q)}(u)<d^{(q)}(v)$, then the graph that arises from $G_{0}$ by removing $u$ and duplicating $v$ has no clique of order $p$ but $\sharp \omega^{(q)}\left(G_{0}\right)-d^{(q)}(u)+d^{(q)}(v)>$ $\sharp \omega^{(q)}(G)$ cliques of order $q$, contradicting the choice of $G$. Hence, by symmetry, we may assume that $d^{(q)}(u) \geq d^{(q)}(v), d^{(q)}(w)$. Now, since the edge $v w$ belongs to some clique of order $q$ in $G_{0}$, the graph that arises from $G_{0}$ by removing $v$ and $w$, and triplicating $u$ has no clique of order $p$ but $\sharp \omega^{(q)}\left(G_{0}\right)+2 d^{(q)}(u)-d^{(q)}(v)-d^{(q)}(w)+1>\sharp \omega^{(q)}(G)$ cliques of order $q$, contradicting the choice of G.

Since $G_{0}$ has no clique of order $p$, the multipartite graph $G_{0}$ has $p-1$ (possibly empty) partite sets $V_{1}, \ldots, V_{p-1}$, of orders $n_{1} \geq \ldots \geq n_{p-1}$, respectively. Since $\sharp \omega^{(q)}\left(G_{0}\right)>0$, the graph $G_{0}^{\prime}=$ $G_{0}-\left(V_{1} \cup V_{p-1}\right)$ has a clique of order $q-2$, that is, $\sharp \omega^{(q-2)}\left(G_{0}^{\prime}\right)>0$. If $n_{1} \geq n_{p-1}+2$, then $G_{0}$ has

$$
n_{1} n_{p-1} \sharp \omega^{(q-2)}\left(G_{0}^{\prime}\right)+\left(n_{1}+n_{p-1}\right) \sharp \omega^{(q-1)}\left(G_{0}^{\prime}\right)+\sharp \omega^{(q)}\left(G_{0}^{\prime}\right)
$$

cliques of order $q$, while the graph that arises from $G_{0}$ by moving one vertex from $V_{i}$ to $V_{j}$ has

$$
\left(n_{1}-1\right)\left(n_{p-1}+1\right) \sharp \omega^{(q-2)}\left(G_{0}^{\prime}\right)+\left(n_{1}-1+n_{p-1}+1\right) \sharp \omega^{(q-1)}\left(G_{0}^{\prime}\right)+\sharp \omega^{(q)}\left(G_{0}^{\prime}\right)
$$

cliques of order $q$. Since $\sharp \omega^{(q-2)}\left(G_{0}^{\prime}\right)>0$ and $\left(n_{1}-1\right)\left(n_{p-1}+1\right)>n_{1} n_{p-1}$, this contradicts the choice of $G$. Hence, we obtain $\left|n_{i}-n_{j}\right| \leq 1$ for every $1 \leq i \leq j \leq p-1$, which implies $G_{0}=T_{p-1}(n)$. Since $n \geq p$, all $p-1$ partite sets of $G_{0}$ are non-empty. Therefore, adding any non-edge of $G_{0}$ to $G_{0}$ results in a graph that has a clique of order $p$, which implies $G=G_{0}$, and completes the proof.

A vertex of degree at most 1 is an endvertex, and a neighbor of an endvertex is a support vertex.

Proof of Theorem 3. Within this proof, we call a tree special if it arises by subdividing $n-\alpha-1$ edges of $K_{1, \alpha}$ once. Suppose, for a contradiction, that the theorem is false, and let $n$ be the smallest order for which it fails. Let $T$ be a tree of order $n$ and independence number $\alpha$ such that

- either $\sharp \alpha(T)$ does not satisfy (4) ,

- or $\sharp \alpha(T)$ satisfies (4) with equality but $T$ is not special.

It is easy to see that $T$ is not special and has diameter at least 3 , which implies $\frac{n}{2} \leq \alpha \leq n-2$. We root $T$ at an endvertex of a longest path in $T$. Let $y$ be the parent of an endvertex of maximum depth in $T$, let $x_{1}, \ldots, x_{k}$ be the children of $y$, and let $z$ be the parent of $y$.

The tree $T^{\prime}=T-\left\{x_{1}, \ldots, x_{k}, y\right\}$ has order $n^{\prime}=n-k-1$ and independence number $\alpha^{\prime}=\alpha-k$.

First, we assume that $k \geq 2$. In this case, every maximum independent set in $T$ contains $\left\{x_{1}, \ldots, x_{k}\right\}$, and the choice of $n$ implies

$$
\begin{aligned}
& \sharp \alpha(T) \quad=\quad \sharp \alpha\left(T^{\prime}\right) \\
& \text { (4) } 2^{n^{\prime}-\alpha^{\prime}-1}+1 \\
& \begin{array}{ll}
= & 2^{n-\alpha-2}+1 \\
\alpha \leq n-2 & 2^{n-\alpha-1}
\end{array}
\end{aligned}
$$

Now, if $\sharp \alpha(T)=2^{n-\alpha-1}$, then 
- equality holds in (7), which implies $2(\alpha-k)=2 \alpha^{\prime}=n^{\prime}=n-k-1$, and

- equality holds in (8), which implies $\alpha=n-2$.

These equations imply $k=n-3, \alpha^{\prime}=1$, and $n^{\prime}=2$, that is, $T^{\prime}$ is $K_{2}$. We obtain the contradiction, that $T$ arises by sudvidiving one edge of $K_{1, \alpha}$, that is, $T$ is special. Hence, we may assume that $k=1$.

Since the number of maximum independent sets in $T$ that contain $y$ is less or equal than the number of maximum independent sets in $T$ that contain $x$, we obtain $\sharp \alpha(T) \leq 2 \sharp \alpha\left(T^{\prime}\right)$, and $\sharp \alpha(T)<2 \sharp \alpha\left(T^{\prime}\right)$ if some maximum independent set in $T^{\prime}$ that contain $z$.

First, we assume that $2 \alpha=n$ and that $T^{\prime}$ is not special. Since $2 \alpha^{\prime}=2 \alpha-2=n-2=n^{\prime}$, the tree $T^{\prime}$ is a bipartite graph whose partite sets both have order exactly $\alpha^{\prime}$. This implies that some maximum independent set in $T^{\prime}$ contains $z$, and the choice of $n$ implies the contradiction

$$
\sharp \alpha(T)<2 \sharp \alpha\left(T^{\prime}\right) \stackrel{(4)}{\leq} 2 \cdot 2^{n^{\prime}-\alpha^{\prime}-1}=2^{n-\alpha-1} .
$$

Next, we assume that $2 \alpha=n$ and that $T^{\prime}$ is special. There are only three possibilities for the structure of $T$ illustrated in Figure 1 together with the resulting values of $\sharp \alpha$.
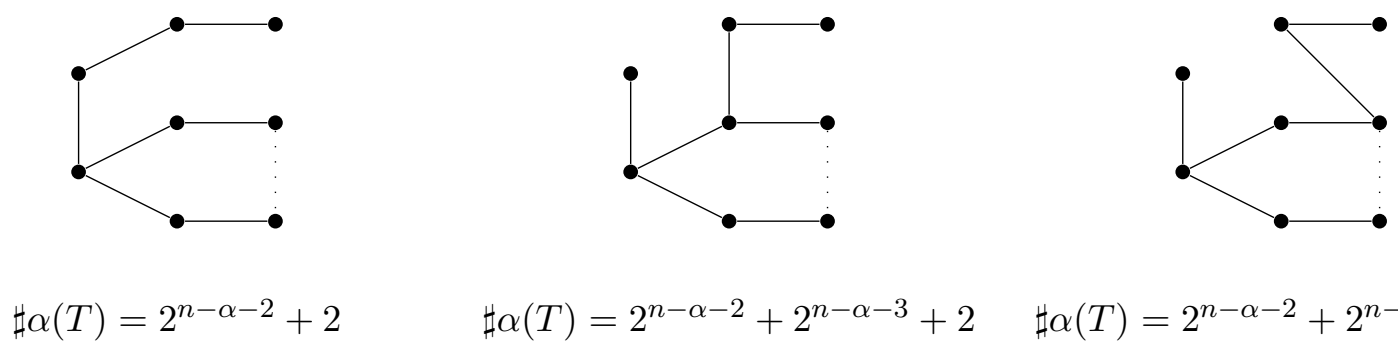

$$
\sharp \alpha(T)=2^{n-\alpha-2}+2
$$$$
\sharp \alpha(T)=2^{n-\alpha-2}+2^{n-\alpha-3}+2 \quad \sharp \alpha(T)=2^{n-\alpha-2}+2^{n-\alpha-3}+1
$$

Figure 1: Three possibilities for the structure of $T$.

In all three cases, we have $n-\alpha-2 \geq 1$, because otherwise either $T$ would be special or the configuration would not be possible. In the first and third case, this already implies a contradiction, because $2^{n-\alpha-2}+2 \leq 2^{n-\alpha-2}+2^{n-\alpha-3}+1 \leq 2^{n-\alpha-1}$. In the second case, we obtain $n-\alpha-2 \geq 2$, because $T$ is not special. Thus, also in this case, we obtain a contradiction, because $2^{n-\alpha-2}+2^{n-\alpha-3}+2 \leq$ $2^{n-\alpha-1}$.

Finally, we assume that $2 \alpha>n$. Since $2 \alpha^{\prime}>n^{\prime}$, the choice of $n$ implies

$$
\begin{aligned}
\sharp \alpha(T) & \leq 2 \sharp \alpha\left(T^{\prime}\right) \\
& \stackrel{\text { (4) }}{\leq} 2 \cdot 2^{n^{\prime}-\alpha^{\prime}-1} \\
& =2^{n-\alpha-1} .
\end{aligned}
$$

Now, if $\sharp \alpha(T)=2^{n-\alpha-1}$, then

- equality holds in (9), which implies that no maximum independent set in $T^{\prime}$ contains $z$, and

- equality holds in (10), which implies that $T^{\prime}$ is special.

Since the only vertex of $T^{\prime}$ that does not belong to some maximum independent set in $T^{\prime}$ is the unique vertex of degree more than 2 in $T^{\prime}$, we obtain the contradiction that $T$ is special, which completes the proof.

Proof of Theorem 因. Suppose, for a contradiction, that the theorem is false, and let $n$ be the smallest order for which it fails. Let $T$ be a subcubic tree of order $n$ and independence number $\alpha=\frac{n}{2}$ such that $\sharp \alpha(T)$ is as large as possible. Note that $n$ is necessarily even.

If $A$ and $B$ are the two partite sets of the bipartite graph $T$, then $\alpha=\frac{n}{2}$ implies $|A|=|B|=\frac{n}{2}$. Furthermore, since $A$ and $B$ are both maximum independent sets in $T$, the neighborhood $N_{T}(S)$ of 
every subset $S$ of $A$ is at least as large as $S$, which, by Hall's theorem [4], implies that $T$ has a perfect matching $M$. If $n \in\{2,4\}$, then $T=T(\alpha)$ follows immediately. Hence, we may assume that $n \geq 6$.

Let the tree $\tilde{T}$ arise from $T$ by contracting all edges in $M$. Let $e_{1} \ldots e_{p}$ be a longest path in $\tilde{T}$. Since $n \geq 6$, we have $p \geq 3$. Let $e_{i}=u_{i} v_{i}$ for $i \in[3]$. By symmetry, we may assume that $u_{2} u_{3}$ is the (unique) edge between $e_{2}$ and $e_{3}$. By the choice of $P$, all neighbors of $e_{2}$ in $\tilde{T}$ that are distinct from $e_{3}$ are endvertices of $\tilde{T}$. Since $T$ has maximum degree at most 3 , the set $N_{\tilde{T}}\left(e_{2}\right) \backslash\left\{e_{3}\right\}$ contains

- $d_{1} \leq 1$ edges $e$ of $T$ such that $u_{2}$ has a neighbor in $e$, and

- $d_{2} \leq 2$ edges $e$ of $T$ such that $v_{2}$ has a neighbor in $e$.

Since $e_{1}$ is one of the edges counted by $d_{1}+d_{2}$, we obtain

$$
\left(d_{1}, d_{2}\right) \in\{(0,1),(0,2),(1,1),(1,2),(1,0)\} .
$$
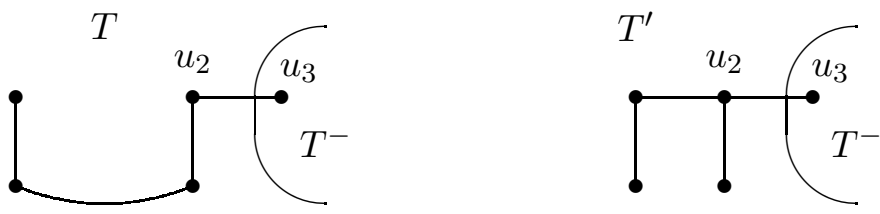

$$
\sharp \alpha(T)=\sharp \alpha_{\epsilon}^{-}+3 \sharp \alpha_{\notin}^{-} \quad<\quad \sharp \alpha\left(T^{\prime}\right)=2 \sharp \alpha_{\epsilon}^{-}+3 \sharp \alpha_{\notin}^{-}
$$
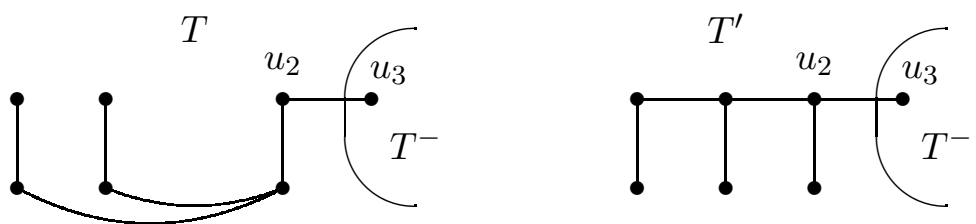

$$
\sharp \alpha(T)=\sharp \alpha_{\epsilon}^{-}+5 \sharp \alpha_{\notin}^{-} \quad<\quad \sharp \alpha\left(T^{\prime}\right)=3 \sharp \alpha_{\epsilon}^{-}+5 \sharp \alpha_{\notin}^{-}
$$
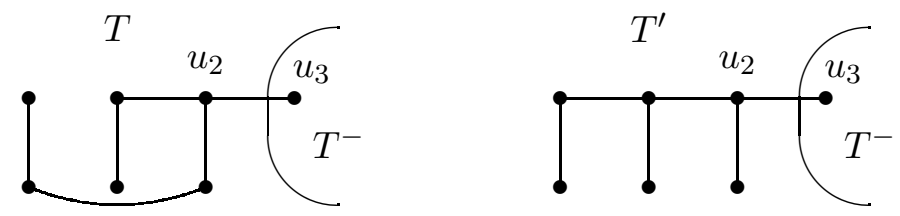

$$
\sharp \alpha(T)=2 \sharp \alpha_{\epsilon}^{-}+4 \sharp \alpha_{\notin}^{-} \quad<\quad \sharp \alpha\left(T^{\prime}\right)=3 \sharp \alpha_{\epsilon}^{-}+5 \sharp \alpha_{\notin}^{-}
$$
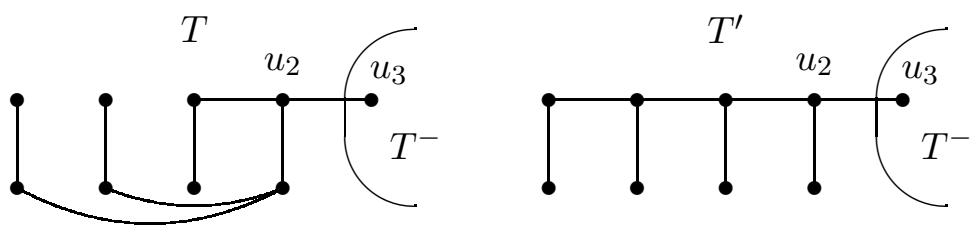

$$
\sharp \alpha(T)=2 \sharp \alpha_{\epsilon}^{-}+6 \sharp \alpha_{\notin}^{-} \quad<\quad \sharp \alpha\left(T^{\prime}\right)=5 \sharp \alpha_{\epsilon}^{-}+8 \sharp \alpha_{\notin}^{-}
$$

Figure 2: $\left(d_{1}, d_{2}\right) \notin\{(0,1),(0,2),(1,1),(1,2)\}$.

Our next goal is to exclude the first four of these possible values of $\left(d_{1}, d_{2}\right)$. In each case, we construct a subcubic tree $T^{\prime}$ of order $n$ and independence number $\alpha=\frac{n}{2}$ such that $\sharp \alpha\left(T^{\prime}\right)>\sharp \alpha(T)$, contradicting the choice of $T$. Let $T^{-}=T-\bigcup_{e \in N_{\tilde{T}}\left(e_{2}\right) \backslash\left\{e_{3}\right\}} e$. By construction, the tree $T^{-}$still has a perfect matching, which implies $\alpha\left(T^{-}\right)=\frac{n\left(T^{-}\right)}{2}$.

Let

- $\sharp \alpha_{\in}^{-}$be the number of maximum independent sets in $T^{-}$that contain $u_{3}$, and let 
- $\sharp \alpha_{\ddagger}^{-}$be the number of maximum independent sets in $T^{-}$that do not contain $u_{3}$.

Since $\alpha\left(T^{-}\right)=\frac{n\left(T^{-}\right)}{2}$, arguing as above implies that both partite sets of the bipartite graph $T^{-}$are maximum independent sets in $T^{-}$, which implies $\sharp \alpha_{\epsilon}^{-}, \sharp \alpha_{\notin}^{-}>0$. Figure 2 illustrates the construction of $T^{\prime}$ in each case, together with the values of $\sharp \alpha(T)$ and $\sharp \alpha\left(T^{\prime}\right)$.

We conclude that $\left(d_{1}, d_{2}\right)=(1,0)$, which implies that the subcubic tree $T^{\prime}$ has order $n-4$ and independence number $\alpha-2=\frac{n-4}{2}$. Let $T^{\prime \prime}=T-\left\{u_{1}, v_{1}\right\}$. The subcubic tree $T^{\prime \prime}$ has order $n-2$ and independence number $\alpha-1=\frac{n-2}{2}$. Therefore, by the choice of $n$, we obtain

$$
\begin{aligned}
\sharp \alpha(T) & =2 \sharp \alpha_{\epsilon}^{-}+3 \sharp \alpha_{\notin}^{-} \\
& =\left(\sharp \alpha_{\epsilon}^{-}+2 \sharp \alpha_{\notin}^{-}\right)+\left(\sharp \alpha_{\epsilon}^{-}+\sharp \alpha_{\notin}^{-}\right) \\
& =\sharp \alpha\left(T^{\prime \prime}\right)+\sharp \alpha\left(T^{\prime}\right) \\
& \leq f(\alpha-1+2)+f(\alpha-2+2) \\
& =f(\alpha+2),
\end{aligned}
$$

that is, $\sharp \alpha(T) \leq f(\alpha+2)$. Furthermore, if $\sharp \alpha(T)=f(\alpha+2)$, then equality holds in (11), which, by the choice of $n$, implies $T^{\prime}=T(\alpha-2)$ and $T^{\prime \prime}=T(\alpha-1)$, and, hence, $T=T(\alpha)$. This contradiction completes the proof.

Proof of Theorem 5. Suppose, for a contradiction, that the theorem is false, and let $n$ be the smallest order for which it fails. Let $T$ be a subcubic tree of order $n$ and independence number $\alpha$. Let $u$ be an endvertex of a longest path $P$ in $T$. By the choice of $n$, the path $P$ has order at least 3 . Let $v$ be the neighbor of $u$, and let $w$ be the neighbor of $v$ on $P$ that is distinct from $u$. The subcubic tree $T^{\prime}=T-\left(N_{T}[v] \backslash\{w\}\right)$ has order $n-d_{T}(v)$ and independence number $\alpha-\left(d_{T}(v)-1\right)$. By the choice of $n$, we obtain

$$
\begin{aligned}
\alpha & =\alpha\left(T^{\prime}\right)+\left(d_{T}(v)-1\right) \\
& \leq \frac{2 n\left(T^{\prime}\right)+1}{3}+\left(d_{T}(v)-1\right) \\
& =\frac{2\left(n-d_{T}(v)\right)+1}{3}+\left(d_{T}(v)-1\right) \\
& =\frac{2 n+1}{3}-\frac{3-d_{T}(v)}{3} \\
& \leq \frac{2 n+1}{3},
\end{aligned}
$$

which implies (6). Now, equality in (6) implies equality in (12) and (13). By the choice of $n$, the tree $T^{\prime}$ arises from $K_{1}$ by iteratively attaching $P_{3}$ s, and that $v$ has degree 3 . Hence, also $T$ arises from $K_{1}$ by iteratively attaching $P_{3}$ s. The uniqueness of the maximum independent set follows easily by an inductive argument exploiting the constructive characterization of $T$. This completes the proof.

Proof of Theorem 6. Suppose, for a contradiction, that the theorem is false, and let $n$ be the smallest order for which it fails. Let $T$ be a subcubic tree of order $n$ and independence number $\alpha$ such that $\sharp \alpha(T)$ is as large as possible.

Claim 1. The tree $T$ contains a path of length at least 3.

Proof of Claim 1. Suppose, for a contradiction, that $T$ is a star $K_{1, n-1}$.

If $n=1$, then

$$
\sharp \alpha(T)=1=\left(\frac{1+\sqrt{5}}{2}\right)^{2-3+1},
$$

if $n=2$, then

$$
\sharp \alpha(T)=2<2.618 \approx\left(\frac{1+\sqrt{5}}{2}\right)^{4-3+1},
$$


if $n=3$, then

$$
\sharp \alpha(T)=1<1.618 \approx\left(\frac{1+\sqrt{5}}{2}\right)^{6-6+1},
$$

and, if $n=4$, then

$$
\sharp \alpha(T)=1=\left(\frac{1+\sqrt{5}}{2}\right)^{8-9+1} .
$$

In each case, we obtain a contradiction to the choice of $n$ and $T$.

Let $u v w x \ldots r$ be a longest path in $T$, and consider $T$ as rooted in $r$. For a vertex $z$ of $T$, let $V_{z}$ be the set that contains $z$ and all its descendants.

Claim 2. $d_{T}(v)=2$

Proof of Claim Q Suppose, for a contradiction, that $d_{T}(v)=3$. Note that every maximum independent set in $T$ contains both children of $v$ but not $v$. Hence, the subcubic tree $T^{\prime}=T-V\left(T_{v}\right)$ has order $n-3$ and independence number $\alpha-2$, and satisfies $\sharp \alpha(T)=\sharp \alpha\left(T^{\prime}\right)$. By the choice of $n$, we obtain

$$
\sharp \alpha(T)=\sharp \alpha\left(T^{\prime}\right) \leq\left(\frac{1+\sqrt{5}}{2}\right)^{2 \cdot(n-3)-3 \cdot(\alpha-2)+1}=\left(\frac{1+\sqrt{5}}{2}\right)^{2 n-3 \alpha+1},
$$

which contradicts the choice of $T$.

Claim 3. $w$ is not a support vertex.

Proof of Claim 3. Suppose, for a contradiction, that $w$ is a support vertex. The subcubic tree $T^{\prime}=$ $T-V\left(T_{v}\right)$ has order $n-2$ and independence number $\alpha-1$, while the subcubic tree $T^{\prime \prime}=T-V\left(T_{w}\right)$ has order $n-4$ and independence number $\alpha-2$. Since there are $\sharp \alpha\left(T^{\prime}\right)$ maximum independent sets in $T$ that contain $u$, and $\sharp \alpha\left(T^{\prime \prime}\right)$ maximum independent sets in $T$ that do not contain $u$, the choice of $n$ implies

$$
\begin{aligned}
\sharp \alpha(T) & =\sharp \alpha\left(T^{\prime}\right)+\sharp \alpha\left(T^{\prime \prime}\right) \\
& \leq\left(\frac{1+\sqrt{5}}{2}\right)^{2 \cdot(n-2)-3 \cdot(\alpha-1)+1}+\left(\frac{1+\sqrt{5}}{2}\right)^{2 \cdot(n-4)-3 \cdot(\alpha-2)+1} \\
& =\left(\frac{1+\sqrt{5}}{2}\right)^{2 n-3 \alpha+1}\left(\left(\frac{1+\sqrt{5}}{2}\right)^{-1}+\left(\frac{1+\sqrt{5}}{2}\right)^{-2}\right) \\
& =\left(\frac{1+\sqrt{5}}{2}\right)^{2 n-3 \alpha+1}
\end{aligned}
$$

which contradicts the choice of $T$.

Claim 4. $d_{T}(w)=2$.

Proof of Claim 4. Suppose, for a contradiction, that $w$ has a child $v^{\prime}$ distinct from $v$. By Claims 2 and 3. the vertex $v^{\prime}$ has exactly one child $u^{\prime}$, which is an endvertex. The subcubic tree $T^{\prime}=T-\left\{u, v, u^{\prime}, v^{\prime}\right\}$ has order $n-4$ and independence number $\alpha-2$. Since for every maximum independent set $I^{\prime}$ of $T^{\prime}$ that does not contain $w$, we have $x \in I^{\prime}$, and $\left(I^{\prime} \backslash\{x\}\right) \cup\{w\}$ is a maximum independent set in $T^{\prime}$ that contains $w$, there are at most $\frac{\sharp \alpha\left(T^{\prime}\right)}{2}$ maximum independent sets in $T^{\prime}$ that do not contain $w$, and at least $\frac{\sharp \alpha\left(T^{\prime}\right)}{2}$ maximum independent sets in $T^{\prime}$ that contain $w$. A maximum independent set in $T^{\prime}$ that contains $w$ can only be extended in a unique way to a maximum independent set in $T$, while a maximum independent set in $T^{\prime}$ that does not contain $w$ can be extended in four different ways to a 
maximum independent set in $T$. Since all maximum independent sets in $T$ are of one of these types, the choice of $n$ implies

$$
\begin{aligned}
\sharp \alpha(T) & \leq 4 \cdot \frac{\sharp \alpha\left(T^{\prime}\right)}{2}+\frac{\sharp \alpha\left(T^{\prime}\right)}{2} \\
& \leq \frac{5}{2} \cdot\left(\frac{1+\sqrt{5}}{2}\right)^{2 \cdot(n-4)-3 \cdot(\alpha-2)+1} \\
& =\frac{5}{2} \cdot\left(\frac{1+\sqrt{5}}{2}\right)^{-2}\left(\frac{1+\sqrt{5}}{2}\right)^{2 n-3 \alpha+1} \\
& <\left(\frac{1+\sqrt{5}}{2}\right)^{2 n-3 \alpha+1}
\end{aligned}
$$

using $\frac{5}{2}<\left(\frac{1+\sqrt{5}}{2}\right)^{2}$, which contradicts the choice of $T$.

Since $\sharp \alpha\left(P_{4}\right)=3<\left(\frac{1+\sqrt{5}}{2}\right)^{2 \cdot 4-3 \cdot 2+1}$ we may assume that $x$ has a parent $y$.

Claim 5. $x$ is not a support vertex.

Proof of Claim 5. Suppose, for a contradiction, that $x$ has a child $w^{\prime}$ that is an endvertex. The subcubic tree $T^{\prime}=T-\{u, v, w\}$ has order $n-3$ and independence number $\alpha-2$. Every maximum independent set $I$ of $T$ contains $u, w$, and $w^{\prime}$, and $I \backslash\{u, w\}$ is a maximum independent set in $T^{\prime}$. By the choice of $n$, this implies

$$
\sharp \alpha(T) \leq \sharp \alpha\left(T^{\prime}\right) \leq\left(\frac{1+\sqrt{5}}{2}\right)^{2 \cdot(n-3)-3 \cdot(\alpha-2)+1}=\left(\frac{1+\sqrt{5}}{2}\right)^{2 n-3 \alpha+1},
$$

which contradicts the choice of $T$.

Claim 6. $x$ has no child that is a support vertex.

Proof of Claim 6. Suppose, for a contradiction, that $x$ has a child $w^{\prime}$ that is a support vertex. If $w^{\prime}$ has two children that are endvertices, then arguing as in the proof of Claim 2 yields a contradiction. If $w^{\prime}$ has a child that is not an endvertex, then $d_{T}\left(w^{\prime}\right)=3$, which leads to a similar contradiction as in the proof of Claim 4. Hence, $w^{\prime}$ has a unique child $v^{\prime}$, which is an endvertex. The subcubic tree $T^{\prime}=T-V\left(T_{x}\right)$ has order $n-6$ and independence number $\alpha-3$. A maximum independent set $I^{\prime}$ of $T^{\prime}$ can be extended in at most four different ways to a maximum independent set in $T: I^{\prime} \cup\left\{u, v^{\prime}, x\right\}$, $I^{\prime} \cup\left\{v, v^{\prime}, x\right\}, I^{\prime} \cup\left\{u, w, w^{\prime}\right\}$ and $I^{\prime} \cup\left\{u, v^{\prime}, w\right\}$. Since all maximum independent sets in $T$ are of such a form, the choice of $n$ implies

$$
\sharp \alpha(T) \leq 4 \sharp \alpha\left(T^{\prime}\right) \leq 4\left(\frac{1+\sqrt{5}}{2}\right)^{2 \cdot(n-6)-3 \cdot(\alpha-3)+1}<\left(\frac{1+\sqrt{5}}{2}\right)^{2 n-3 \alpha+1},
$$

using $4<\left(\frac{1+\sqrt{5}}{2}\right)^{3}$, which contradicts the choice of $T$.

Claim 7. $d_{T}(x)=2$.

Proof of Claim 7. Suppose, for a contradiction, that $x$ has a child $w^{\prime}$ distinct from $w$. By Claims 5 and 6, $w^{\prime}$ has a child $v^{\prime}$ that has a child $u^{\prime}$. By Claims 2 and 4, $d_{T}\left(w^{\prime}\right)=d_{T}\left(v^{\prime}\right)=2$. The subcubic tree $T^{\prime}=T-V\left(T_{x}\right)$ has order $n-7$ and independence number $\alpha-4$. Note that every maximum 
independent set in $T^{\prime}$ can be extended in a unique way to a maximum independent set in $T$, and that the maximum independent sets in $T$ are exactly those sets. Hence, by the choice of $n$, we obtain

$$
\sharp \alpha(T) \leq \sharp \alpha\left(T^{\prime}\right) \leq\left(\frac{1+\sqrt{5}}{2}\right)^{2 \cdot(n-7)-3 \cdot(\alpha-4)+1}<\left(\frac{1+\sqrt{5}}{2}\right)^{2 n-3 \alpha+1} .
$$

By the above claims, we know that $d_{T}(v)=d_{T}(w)=d_{T}(x)=2$. Let $T^{\prime}=T-V\left(T_{x}\right), T_{1}=$ $T-\{v u\}+\{x u\}$, and $T^{\prime \prime}=T_{1}-\{v, w\}$. Clearly, all these trees are subcubic.

A maximum independent set in $T^{\prime}$ that contains $y$ can only be extended in a unique way to a maximum independent set in $T$, and all maximum independent set in $T$ that contain $y$ are of that form. A maximum independent set $I^{\prime}$ of $T^{\prime}$ that does not contain $y$ can be extended to a maximum independent set $I$ of $T$ in three ways, $I^{\prime} \cup\{u, w\}, I^{\prime} \cup\{u, x\}$ and, $I^{\prime} \cup\{v, x\}$, and every maximum independent set in $T$ that does not contain $y$ is of that form.

Similarly, a maximum independent set in $T^{\prime}$ that contains $y$ can be extended to a maximum independent set in $T_{1}$ in two different ways, and all maximum independent set in $T_{1}$ that contain $y$ are of that form. A maximum independent set $I^{\prime}$ of $T^{\prime}$ that does not contain $y$ can be extended to a maximum independent set $I_{1}$ of $T_{1}$ in three ways, $I^{\prime} \cup\{u, w\}, I^{\prime} \cup\{u, v\}$ and, $I^{\prime} \cup\{v, x\}$, and every maximum independent set in $T_{1}$ that does not contain $y$ is of that form. Arguing as in the proof of Claim 3, we obtain

$$
\sharp \alpha(T) \leq \sharp \alpha\left(T_{1}\right)=\sharp \alpha\left(T^{\prime}\right)+\sharp \alpha\left(T^{\prime \prime}\right) \leq\left(\frac{1+\sqrt{5}}{2}\right)^{2 n-3 \alpha+1} .
$$

This final contradiction completes the proof.

\section{References}

[1] M. Aigner and G.M. Ziegler, Proofs from The Book, Springer-Verlag, Berlin, 3rd edition, 2004.

[2] J.D. Alvarado, S. Dantas, E. Mohr, and D. Rautenbach, On the maximum number of minimum dominating sets in forests, arXiv:1804.00158.

[3] T. Derikvand and M.R. Oboudi, On the number of maximum independent sets of graphs, Transactions on Combinatorics 3 (2014) 29-36.

[4] P. Hall, On representatives of subsets, Journal of the London Mathematical Society 10 (1935) 26-30.

[5] M.-J. Jou and G.J. Chang, The number of maximum independent sets in graphs, Taiwanese Journal of Mathematics 4 (2000) 685-695.

[6] K.M. Koh, C.Y Goh, and F.M. Dong, The maximum number of maximal independent sets in unicyclic connected graphs, Discrete Mathematics 308 (2008) 3761-3769.

[7] J.W. Moon and L. Moser, On cliques in graphs, Israel Journal of Mathematics 3 (1965) 23-28.

[8] J.M. Nielsen, On the number of maximal independent sets in a graph, BRICS Report Series RS02-15 (2002).

[9] S. Roman, The maximum number of $q$-cliques in a graph with no $p$-clique, Discrete Mathematics 14 (1976) 365-371.

[10] P. Turán, Eine Extremalaufgabe aus der Graphentheorie, Matematikai és Fizikai Lapok 48 (1941) 436-452. 
[11] I. Włoch, Trees with extremal numbers of maximal independent sets including the set of leaves, Discrete Mathematics 308 (2008) 4768-4772.

[12] J. Zito, The structure and maximum number of maximum independent sets in trees, Journal of Graph Theory 15 (1991) 207-221.

[13] A.A. Zykov, On some properties of linear complexes, Matematicheskiư Sbornik. Novaya Seriya 24(66) (1949) 163-188. 